EPJ Web of Conferences 38, 04001 (2012)

DOI: $10.1051 /$ epjconf/20123804001

(c) Owned by the authors, published by EDP Sciences, 2012

\title{
Probing the pairing interaction through two-neutron transfer reactions
}

\author{
M. Grasso ${ }^{1, \text { a }}$, D. Beaumel ${ }^{1}$, E. Khan ${ }^{1}$, D. Lacroix ${ }^{2}$, J. Margueron ${ }^{1}$, and A. Vitturi ${ }^{3}$ \\ 1 Institut de Physique Nucléaire, IN2P3-CNRS, Université Paris-Sud, F-91406 Orsay Cedex, France \\ 2 Grand Accélérateur National d'Ions Lourds (GANIL), CEA/DSM-CNRS/IN2P3, Bvd Henri Becquerel, F-14076 Caen, \\ France \\ 3 Dipartimento di Fisica G. Galilei, via Marzolo 8, I-35131 Padova, Italy; Istituto Nazionale di Fisica Nucleare (INFN), \\ Sezione di Padova, via Marzolo 8, I-35131 Padova, Italy
}

\begin{abstract}
The treatment of the pairing interaction in mean-field-based models is addressed. In particular, the possibility to use pair transfers as a tool to better constrain this interaction is discussed. First, pairing interactions with various density dependencies (surface/volume mixing) are used in the microscopic Hartree-FockBogoliubov + quasiparticle random-phase approximation model to generate the form factors to be used in reaction calculations. Cross sections for $(\mathrm{p}, \mathrm{t})$ two-neutron transfer reactions are calculated in the one-step zero-range distorted-wave Born approximation for some Tin isotopes and for incident proton energies from 15 to $35 \mathrm{MeV}$. Three different surface/volume mixings of a zero-range density-dependent pairing interaction are employed in the microscopic calculations and the sensitivity of the cross sections to the different mixings is analyzed. Differences among the three different theoretical predictions are found espacially for the nucleus ${ }^{136} \mathrm{Sn}$ and they are more important at the incident proton energy of $15 \mathrm{MeV}$. We thus indicate (p,t) two-neutron transfer reactions with very neutron-rich $\mathrm{Sn}$ isotopes and at proton energies around $15 \mathrm{MeV}$ as good experimental cases where the surface/volume mixing of the pairing interaction may be probed. In the second part of the manuscript, ground-state to ground-state transitions are investigated. Approximations made to estimate two-nucleon transfer probabilities in ground-state to ground-state transitions and the physical interpretation of these probabilities are discussed. Probabilities are often calculated by approximating both ground states of the initial nucleus $A$ and of the final nucleus $A \pm 2$ by the same quasiparticle vacuum. We analyze two improvements of this approach. First, the effect of using two different ground states with average numbers of particles $A$ and $A \pm 2$ is quantified. Second, by using projection techniques, the role of particle number restoration is analyzed. Our analysis shows that the improved treatment plays a role close to magicity, leading to an enhancement of the pair-transfer probability. In midshell regions, part of the error made by approximating the initial and final ground states by a single vacuum is compensated by projecting onto a good particle number. Surface effects are analyzed by using pairing interactions with a different volume/surface mixing. Finally, a simple expression of the pair-transfer probability is given in terms of occupation probabilities in the canonical basis. We show that, in the canonical basis formulation, surface effects that are visible in the transfer probability are related to the fragmentation of single-particle occupancies close to the Fermi energy. This provides a complementary interpretation with respect to the standard quasiparticle representation where surface effects are generated by the integrated radial profiles of the contributing wave functions.
\end{abstract}

\section{Introduction}

Pairing correlations play an important role in determining the properties of a large number of open-shell superfluid nuclei. The link between Cooper-pair superfluidity in nuclei and cross sections associated to pair-transfer reactions has been extensively discussed in the literature starting from the early work of Broglia and collaborators in the 70 s $[1,2]$. In particular, the relation between the characteristics of the pairing correlations and the transfer probabilities in two-particle transfer reactions has been analyzed [3]. Recently, there is a renewal of interest on experimental 1-nucleon $(1 n)$, 2-nucleon $(2 n)$ and more generally multinucleon transfer channels at bombarding energies close to the Coulomb barrier [4-6]. From the theoretical side, in the last decade, new microscopic calculations have been developed, essentially in the framework of the Hartree-Fock-

\footnotetext{
a e-mail: grasso@ipno.in2p3.fr
}

Bogoliubov (HFB) + quasiparticle random-phase approximation (QRPA) theory to investigate the properties of the $0^{+}[7]$ and $2^{+}$[8] excitation modes associated to $2 n$ addition or removal during transfer reactions. A similar analysis has been performed also within the time-dependent HFB model in the small-amplitude limit [9].

The excitation modes related to the transfer of nucleonic pairs in superfluid nuclei are expected to be strongly sensitive to the specific features of the pairing interaction. It has been recently suggested that the observables related to pairing vibrations could be considered as useful additional constraints in the fitting procedures of phenomenological interactions $[8,10,11]$. In particular, in the currently used zero-range density-dependent interaction, the surface / volume mixing of the interaction can be tuned by modifying the parameter $x$ in the expression

$$
V\left(\mathbf{r}_{1}, \mathbf{r}_{2}\right)=\delta\left(\mathbf{r}_{1}-\mathbf{r}_{2}\right) V_{0}\left[1+x\left(\frac{\rho(\mathbf{R})}{\rho_{0}}\right)^{\alpha}\right],
$$


where $\mathbf{R}=\left(\mathbf{r}_{1}+\mathbf{r}_{2}\right) / 2 ; x=0$ and $x=1$ represent the extreme cases of a pure volume and a pure surface interaction, respectively. Microscopic quasiparticle randomphase approximation (QRPA) calculations for the $0^{+}$[10] and the $2^{+}[8]$ pair-transfer modes have been performed and it has been shown that the transition densities actually depend on the different choices of the pairing interaction in terms of surface/volume mixing. In particular, differences have been found between the two cases of a pure surface interaction and a mixed interaction.

To finally prove that the observables associated to pairing vibrations can indeed guide us toward a deeper comprehension of this specific aspect of the pairing interaction, cross sections have to be evaluated. The analysis is pursued here in this direction. The idea is to perform a calculation of cross sections where the form factor of the transition is evaluated microscopically. The self-consistent microscopic QRPA results are used as structure inputs for the reaction calculation. We consider two-neutron tranfer $(p, t)$ reactions where the Hartree-Fock-Bogoliubov (HFB) + QRPA approach is used to describe the microscopic nuclear structure and the one-step distorted-wave Born approximation (DWBA) is employed for the reaction dynamics. The cross sections in the DWBA are calculated using the Distorted Waves University of Colorado Kunz 4 (DWUCK4) code [12].

In the recent Ref. [13], the effects of the surface/volume nature of the pairing interaction on $2 n$ transfer have been extensively analyzed. The enhancement of transfer probabilities at the surface has been predicted (when a surfacepeaked interaction is used) in the transition from the ground state (GS) of the nucleus with mass $A$ to the GS of the nuclei with masses $A \pm 2$ in $\mathrm{Sn}$ isotopes beyond $N=82$. Similarly to what is done in other recent estimates of the pairtransfer probability using the microscopic HFB approach [4], the strength associated to these transitions has been calculated in Ref. [13] with an approximate formula where only the wave functions of the nucleus $A$ enter. This approximate treatment differs from the original formulation given in Ref. [1] where the components of both nuclei $A$ and $A \pm 2$ appear. Accordingly, in other recent works not based on HFB [14], in the expressions of the two-particle transfer spectroscopic amplitudes the wave functions of the two nuclei $A$ and $A+2$ appear [14]. It is worth mentioning that, even the formulas given in Ref. [1] are approximate expressions and, as far as we know, the underlying approximation needs to be clarified.

One goal of the present work is to discuss different level of approximation used to estimate two-nucleon transition probabilities. In this work we derive the expressions to be used for the two-particle GS $\rightarrow$ GS transfer probabilities in the framework of the HFB model. We compare our results with those obtained with the model of Shimoyama and Matsuo [13] and analyze the differences in mid-shells and at shell closures. Following their work, different values for the surface/volume mixing parameter are used in the pairing interaction and application is made for the chain of Sn isotopes. By using the canonical basis representation of the HFB model we provide a complementary interpretation of surface effects. Finally, we quantify the effect of particle number restoration on two-neutron transfer probabilities.

The article is organized as follows: In Sec. 2 the cross sections for $(p, t)$ transfer reactions are calculated. In Sec.
3 an improved treatment of the transfer transition from the ground state of the initial nucleus to the final state of the final nucleus is addressed. We conclude in Sec. 4.

\section{Cross sections for $(p, t)$ reactions with different surface/volume mixings}

For the excitation modes associated to $(p, t)$ reactions, the transition densities are calculated between the ground state of the nucleus with $A$ nucleons and a state of the nucleus with $A-2$ nucleons. The form factor is obtained by folding the transition density with the interaction between the transferred pair and the residual nucleus. In our model, this interaction is actually chosen of zero-range and this means that the transition densities directly provide the form factors. It is clear that with a zero-range interaction the shapes of the angular distributions can be well defined but the absolute values of the cross sections cannot be evaluated.

The structure calculations to derive the transition densities are performed in this work within the HFB + QRPA framework. Optical potentials have to be provided together with the form factors for the reaction calculations. Phenomenological optical potentials are used here. The optical potential for the entrance channel (interaction between the proton and the nucleus) is constructed with the parameters of Ref. [15] which have been fitted on elastic scattering of protons by nuclei with $A>40$ and with a proton laboratory energy $E_{p}<50 \mathrm{MeV}$. For the exit channel, the optical potential parameters have been fitted on the elastic scattering of the triton [16].

In the DWBAcalculations performed with the DWUCK4 code, a neutron pair of zero angular momentum is transferred from one nucleus to the other. The transitions are calculated for different incident proton energies $E_{p}$ and with the three different pairing interactions. Incident proton energies range from 15 to $35 \mathrm{MeV}$. We have first considered the reaction ${ }^{124} \mathrm{Sn}(p, t){ }^{122} \mathrm{Sn}$. When drip lines are approached, neutron skins become thicker: surface effects and low-density pairing features are thus expected to be typically more important. Owing to this, we have considered also the pair-transfer reaction ${ }^{136} \mathrm{Sn}(p, t){ }^{134} \mathrm{Sn}$ where a more neutron-rich nucleus is involved. With the nextgeneration facilities, it is expected that beams of very neutronrich tin isotopes such as ${ }^{134,136} \mathrm{Sn}$ will be produced with sufficiently high intensity for performing two-nucleon transfer experiments.

We have observed that the profiles of the cross sections corresponding to $x=1$ and $x=0.35$ in Eq. (1) differ at large angles $\left(\Theta_{C M}>70\right.$ degrees) for the more neutron-rich nucleus ${ }^{136} \mathrm{Sn}$. We are aware that measurements are more difficult at large angles because the corresponding cross sections are very low. Nevertheless, this result indicates that very neutron-rich $\mathrm{Sn}$ isotopes may be interesting cases to analyze. On the basis of this first indication, we present our investigation for ${ }^{136} \mathrm{Sn}$ and we show in figure 1 the ratios of the cross sections associated to the first two $0^{+}$transitions at different proton energies. Even if absolute cross sections cannot be calculated within the present reaction model, the ratios of the cross sections related to the first transitions are meaningful quantities to analyze. These ratios are proportional to the ratios of the transition probabilities associated to the 
two transitions and that the proportionality factor is the same independently of the pairing interaction. The comparison of the ratios obtained with different pairing interactions can thus provide interesting predictions about the sensibility of the cross sections to the choice of the pairing interaction. It can be seen that differences exist among the three sets of results and they are more important at the lowest energy of $15 \mathrm{MeV}$, that represents the case where the reaction takes place mostly in the surface region of the nucleus. Hence, we suggest very neutron-rich $\mathrm{Sn}$ isotopes and proton energies around $15 \mathrm{MeV}$ as favorable cases for future $(p, t)$ or $(t, p)$ pair-transfer experiments that can provide a deeper insight into the surface/volume character of the pairing interaction. Performing $(t, p)$ reaction measurements in inverse kinematics is quite more challenging than $(p, t)$, but such reactions would allow one to populate different states of $\mathrm{Sn}$ isotopes that may also represent very favourable cases for pairing studies.

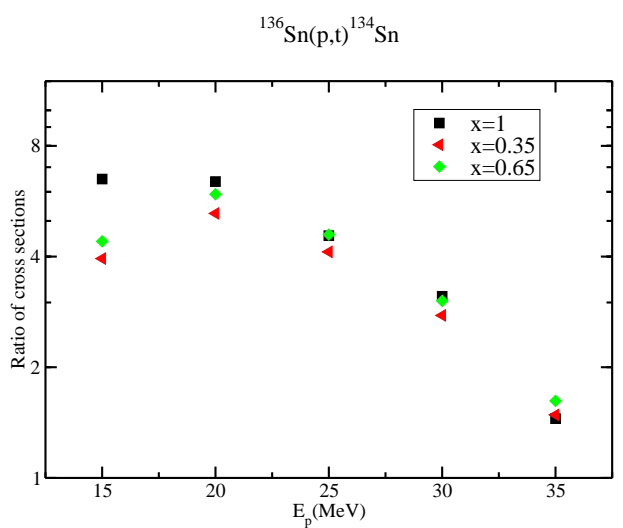

Fig. 1. Ratios of the cross sections associated to the first two $0^{+}$transitions at different proton energies for the reaction ${ }^{136} \mathrm{Sn}(p, t){ }^{134} \mathrm{Sn}$.

\section{Improved treatment of GS $\rightarrow$ GS pair transfer}

In this section, we discuss how to obtain, in the framework of the HFB model, a formulation similar to that advanced in Ref. [1] for the GS $\rightarrow$ GS transitions. This improved formalism with respect to that of Ref. [13] leads to expressions where the wave functions of the initial and the final nucleus appear.

\subsection{Quasiparticle formulation}

We write explicitly the Bogoliubov transformations in the expressions for the amplitudes for the removal and addition transitions. For the removal amplitude this means,

$$
\begin{aligned}
T_{\mathrm{GS}}^{\mathrm{Rem}}(A, r) & =\sum_{n n^{\prime}}\left\langle\mathrm{GS}_{A-2}\right|\left(u_{n}^{A-2}\left(\mathbf{r},-\sigma_{1}\right) \gamma_{n-\sigma_{1}}\right. \\
& \left.+(-1)^{1 / 2-\sigma_{1}} v_{n}^{* A-2}\left(\mathbf{r}, \sigma_{1}\right) \gamma_{n \sigma_{1}}^{\dagger}\right) \\
\left(u_{n^{\prime}}^{A}\left(\mathbf{r}, \sigma_{2}\right) \gamma_{n^{\prime} \sigma_{2}}\right. & +(-1)^{1 / 2+\sigma_{2}} v_{n^{\prime}}^{* A}\left(\mathbf{r},-\sigma_{2}\right) \gamma_{n^{\prime}-\sigma_{2}}^{\dagger}\left|\mathrm{GS}_{A}\right\rangle
\end{aligned}
$$

An analogous expression may be written for the addition amplitude. To explicitly evaluate the matrix elements of the operators $\gamma$ we have to adopt the following approximations:

$$
\begin{aligned}
&\left\langle\mathrm{GS}_{A \pm 2}\left|\gamma_{n, \sigma_{1}}^{\dagger} \gamma_{n^{\prime}, \sigma_{2}}\right| \mathrm{GS}_{A}\right\rangle \sim\left\langle\mathrm{GS}_{A}\left|\gamma_{n, \sigma_{1}}^{\dagger} \gamma_{n^{\prime}, \sigma_{2}}\right| \mathrm{GS}_{A}\right\rangle=\delta_{n n^{\prime}} \delta_{\sigma_{1} \sigma_{2}} \\
&\left\langle\mathrm{GS}_{A \pm 2}\left|\gamma_{n, \sigma_{1}} \gamma_{n^{\prime}, \sigma_{2}}\right| \mathrm{GS}_{A}\right\rangle \sim\left\langle\mathrm{GS}_{A}\left|\gamma_{n, \sigma_{1}} \gamma_{n^{\prime}, \sigma_{2}}\right| \mathrm{GS}_{A}\right\rangle=0 \\
&\left\langle\mathrm{GS}_{A \pm 2}\left|\gamma_{n, \sigma_{1}}^{\dagger} \gamma_{n^{\prime}, \sigma_{2}}^{\dagger}\right| \mathrm{GS}_{A}\right\rangle \sim\left\langle\mathrm{GS}_{A}\left|\gamma_{n, \sigma_{1}}^{\dagger} \gamma_{n^{\prime}, \sigma_{2}}^{\dagger}\right| \mathrm{GS}_{A}\right\rangle=0
\end{aligned}
$$

and thus obtain for the removal and addition amplitudes

$$
\begin{aligned}
& T_{\mathrm{GS}}^{\mathrm{Rem}}(A, r) \sim-\frac{1}{4 \pi r^{2}} \sum_{n l j}(2 j+1) u_{n l j}^{A-2}(r) v_{n l j}^{A}(r), \\
& T_{\mathrm{GS}}^{\mathrm{Add}}(A, r) \sim-\frac{1}{4 \pi r^{2}} \sum_{n l j}(2 j+1) u_{n l j}^{A}(r) v_{n l j}^{A+2}(r) .
\end{aligned}
$$

In these expressions the wave functions of the two nuclei appear as originally used in Ref. [1].

The pair-transfer strengths are given by

$$
\begin{aligned}
& P_{\mathrm{GS}}^{\mathrm{Rem}}(A)=\left|\int d r \sum_{n l j}(2 j+1) u_{n l j}^{A-2}(r) v_{n l j}^{A}(r)\right|^{2}, \\
& P_{\mathrm{GS}}^{\mathrm{Add}}(A)=\left|\int d r \sum_{n l j}(2 j+1) u_{n l j}^{A}(r) v_{n l j}^{A+2}(r)\right|^{2} .
\end{aligned}
$$

One notices also that

$$
P_{\mathrm{GS}}^{\mathrm{Rem}}(A+2)=P_{\mathrm{GS}}^{\mathrm{Add}}(A) .
$$

\subsection{Canonical formalism}

Assuming that the canonical states do not change too much between the nucleus $A$ and $A \pm 2$ (no core polarization due to the addition or removal of two nucleons) the following formula can be obtained:

$$
\begin{aligned}
T_{\mathrm{GS}}^{\mathrm{Add}}(A, \mathbf{r}) & \simeq-\sum_{i>0}\left\langle\mathrm{GS}_{A+2}\left|a_{i}^{\dagger} a_{i}^{\dagger}\right| \mathrm{GS}_{A}\right\rangle\left(\left|\varphi_{i}(\mathbf{r}, \uparrow)\right|^{2}+\left|\varphi_{i}(\mathbf{r}, \downarrow)\right|^{2}\right) \\
& =\sum_{i>0} \sqrt{n_{i}^{A+2}\left(1-n_{i}^{A}\right)}\left(\left|\varphi_{i}(\mathbf{r}, \uparrow)\right|^{2}+\left|\varphi_{i}(\mathbf{r}, \downarrow)\right|^{2}\right)
\end{aligned}
$$

and

$$
T_{\mathrm{GS}}^{\mathrm{Rem}}(A, \mathbf{r})=-\sum_{i>0} \sqrt{\left(1-n_{i}^{A-2}\right) n_{i}^{A}}\left(\left|\varphi_{i}(\mathbf{r}, \uparrow)\right|^{2}+\left|\varphi_{i}(\mathbf{r}, \downarrow)\right|^{2}\right) .
$$

Accordingly, the two-particle addition and removal probabilities read

$$
\begin{aligned}
& P_{\mathrm{GS}}^{\mathrm{Add}}(A)=\left|\sum_{i} \sqrt{n_{i}^{A+2}\left(1-n_{i}^{A}\right)}\right|^{2}=\left|\sum_{i}(2 j+1) \sqrt{n_{n l j}^{A+2}\left(1-n_{n l j}^{A}\right)}\right|^{2}, \\
& P_{\mathrm{GS}}^{\mathrm{Rem}}(A)=\left|\sum_{i} \sqrt{\left(1-n_{i}^{A-2}\right) n_{i}^{A}}\right|^{2}=\left|\sum_{i}(2 j+1) \sqrt{\left(1-n_{n l j}^{A-2}\right) n_{n l j}^{A}}\right|^{2} .
\end{aligned}
$$


The two above equations show that Eq. (7) holds also in the canonical basis formulation. It should be noted that the approximation made to obtain the above expressions slightly differs from the one used in the previous subsection and differences between the two sets of results may actually be expected within the improved formalism.

\subsection{Results and discussion}

Since the difference between removal and addition probabilities is just a shift of nuclei (see Eq. (7)), we consider in what follows only removal probabilities. In figures 2 and 3 the results obtained with the improved expressions for the removal strength are presented for the mixed and the surface interaction, respectively. The canonical basis results (filled circles) are compared with the quasiparticle results (dashed line) and with the results obtained with the simplified formula of Ref. [13] (solid line). In figure 2, we see that the canonical and quasiparticle improved forms give similar results. In particular, these new forms of the pairtransfer probability lead to non-zero values for magic nuclei. It is also worth mentioning that the probability is enhanced in the mid-shell with respect to the corresponding value given by the solid line.

The case of figure 3 is different. We observe some irregularities in the trend of the results which have been obtained with Eq. (5). These irregularities are related to some specific features of the HFB calculations in coordinate representation. It may happen that the fragmentation of the occupations among the discretized quasiparticle states (box boundary conditions with a box radius equal to $20 \mathrm{fm}$ ) is not the same in the nucleus $A \pm 2$ and in the nucleus $A$. When this is the case, some irregularities appear in the transfer strength calculated by making products of functions belonging to the different nuclei $A$ and $A \pm 2$. This situation does not occur when one uses the canonical basis, that displays a smoother behavior when moving from one system to the neighbor one. There irregularities are much more pronounced in the case of surface-peaked interaction. These results indicate that the improved treatment of pair transfer discussed here should be done using a discrete basis instead of the coordinate representation to avoid the irregularities.

We also observe that the differences between the results obtained with the canonical and the quasiparticle formulations are more pronounced in the case of a surface-peaked interaction (figure 2). This may be related to the fact that the artificial irregularities of the quasiparticle results are more important in the case of a surface pairing interaction (the fragmentation of the occupation numbers may vary more strongly from the nucleus $A$ to the nucleus $A \pm 2$ in this case). The slightly different approximation adopted for the ground states of the two nuclei in the two derivations also accounts for these differences.

\section{Effect of particle number conservation on pair transfer probability}

The quasiparticle states which are generally used to estimate pair-transfer probabilities are not eigenstates of the

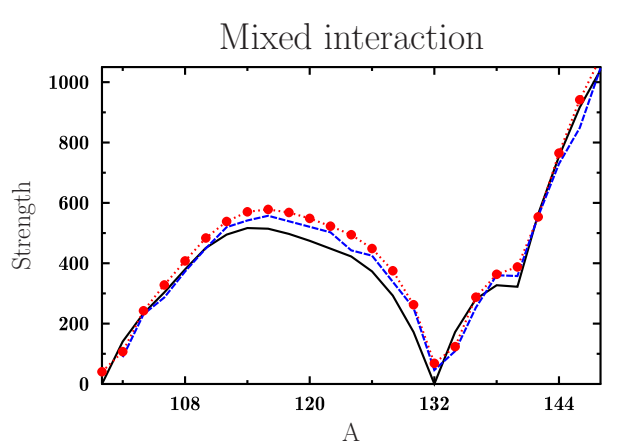

Fig. 2. Comparison of the removal probability obtained with the mixed pairing case (solid line) and the improved expressions in the quasiparticle (dashed curve) and in the canonical (filled circles) formalism.

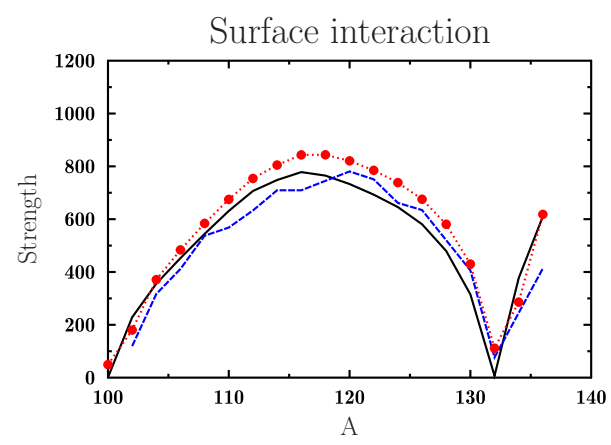

Fig. 3. Same as in figure 2 but for the case of a surface-peaked pairing interaction.

particle number operator. In particular, a state $\left|\mathrm{GS}_{A}\right\rangle$ contains not only components with the correct number of particles, but also components with particle numbers $A \pm 2$, $A \pm 4, \ldots$ inducing spurious contributions to the probabilities. These contributions can be exactly removed using projection techniques. In the following, it is assumed that the ground state of a system with particle number $A$ is written as

$$
\left|\mathrm{GS}_{A}\right\rangle \simeq|A\rangle=P^{A} \prod_{i>0}\left(u_{i}^{A}+v_{i}^{A} a_{i}^{\dagger} a_{i}^{\dagger}\right)|0\rangle
$$

where $P^{A}$ denotes the projector onto the number of particles $A$. The projected state has the particularity that it has the same canonical basis as the original quasiparticle vacuum from which it is constructed. The transition probability accounting for particle number projection can now be approximately estimated by replacing occupation numbers of the quasi-particle state $n_{i}^{A}$ in Eqs. (10) and (11) by the new equivalent occupation numbers, denoted by $\bar{n}_{i}^{A}$, in the projected state, i.e.:

$$
\begin{aligned}
& \tilde{P}_{\mathrm{GS}}^{\mathrm{Add}}=\left|\sum_{i} \sqrt{\bar{n}_{i}^{A+2}\left(1-\bar{n}_{i}^{A}\right)}\right|^{2}, \\
& \tilde{P}_{\mathrm{GS}}^{\mathrm{Rem}}=\left|\sum_{i} \sqrt{\left(1-\bar{n}_{i}^{A-2}\right) \bar{n}_{i}^{A}}\right|^{2} .
\end{aligned}
$$

In figures 4 and 5 , the effect of particle number conservation on the estimation of the transition probabilities is illustrated for mixed and pure surface pairing, respectively. 
In these figures, we clearly see a very interesting and unexpected effect: at mid-shells, the projection actually tends to cancel out the effect of using the occupation numbers of the $A$ and $A \pm 2$ nuclei in the improved formulation. This reduction is due to the fact that the projection tends to reduce the fragmentation around the Fermi energy compared to the non-projected state and the single-particle fragmentation directly affects the two particles transfer probability [17]. The pairing strength gets again closer to the probability obtained with the approximation of Ref. [13]. Note that the fact that the projected and non-projected curves are very close from each other at the mid-shell is purely accidental. Close to magicity, projection has little effect and the results remain unchanged with respect to the improved treatment of the transfer probability. We mention that in Ref. [13], in order to have a non zero probability at shell closures, the transfer probability is calculated in these cases with the particle-particle random-phase approximation. Non-zero values are obtained here within a different approach based on a unified approach suited to treat all the nuclei, both at shell closures and in mid-shell regions.

It is finally worth mentioning that the projection is made here after variation (PAV). If variation is made after projection (VAP), it is anticipated that the strength will increase compared to the PAV both at mid-shell and at shell-closure due to the enhanced fragmentation of single-particle states (see Ref. [18]) and due to the absence of pairing threshold anomaly, leading to pairing even in close shell nuclei.

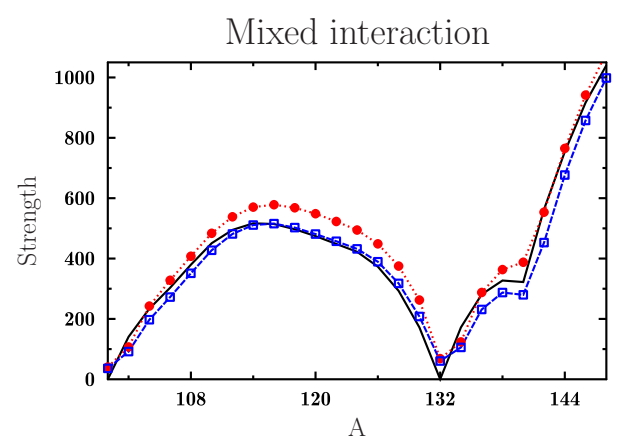

Fig. 4. Removal transfer probability obtained accounting for particle number conservation (open square) and compared to the improved (Sec. 4) (filled circles) and more approximated (Sec. 3) expressions (solid line) for the mixed interaction.

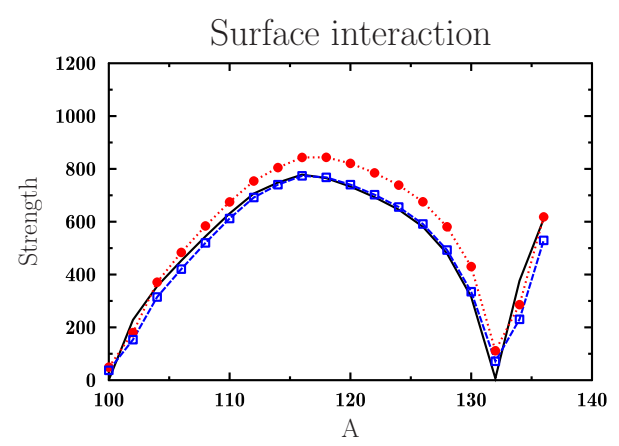

Fig. 5. Same as in figure 4 for the case of pure surface pairing interaction.

\section{Conclusions}

We have analyzed two-neutron transfer reactions for Tin isotopes. In the first part of the manuscript we have considered transfers from the ground state of the initial nucleus to an excited state of the final nucleus. Cross sections have been evaluated by using microscopic form factors coming from HFB + QRPA calculations. An interesting case seems to be the reaction ${ }^{136} \mathrm{Sn}(p, t){ }^{134} \mathrm{Sn}$ where a very neutronrich nucleus is involved. For some values of the energy of the proton, discrepancies at large angles $\left(\Theta_{C M}>70 \mathrm{de}-\right.$ grees) are found in the position of the minima between the pure-surface case and the mixed-interaction case (the two mixed interactions lead to very similar results). This is a first qualitative indication that suggests that this case can be interesting to be explored experimentally. We have compared the ratios of the cross sections associated to the first two $0^{+}$transitions at different proton energies. Sizeable differences among the three theoretical predictions are found especially at the lowest proton energy of $15 \mathrm{MeV}$. New-generation accelerators should allow soon to produce ${ }^{134,136} \mathrm{Sn}$ beams with sufficiently high intensity. The conclusion is that pair-transfer reactions for a very neutronrich $\mathrm{Sn}$ isotope and at proton energies around $15 \mathrm{MeV}$ (reactions in the surface region of the nucleus) may be good experimental cases where the surface/volume nature of the pairing interaction can be elucidated.

In the second part of the manuscript transitions from the ground state of the initial nucleus to the ground state of the final nucleus are considered. The possibility to improve the description of pair transfer probabilities in a meanfield model is then discussed. Two improvements are introduced: (i) the use of two different quasi-particle vacuum for the initial and final ground states; (ii) the possibility to exactly restore the proper number of particles in the entrance channel. It is shown that these refinements are important especially close to the magicity where a nonzero probability is found contrary to the simplest method. It turns out that the enhancement observed in the mid-shell by taking two different ground states is partially compensated by the particle number restoration. We can thus finally conclude that the simple formula used in Ref. [13], although approximated, can still provide good results at mid-shells. We remind however that the projection is performed here a posteriori. If variation after projection is made, additional correlations are expected to appear especially close to magic numbers [18]. This open new perspectives and could be an interesting subject for future investigations.

\section{References}

1. R.A. Broglia, O. Hansen, and C. Riedel, Adv. Nucl. Phys. 6, 287 (1973)

2. G. Ripka and R. Padjen, Nucl. Phys. A 132, 489 (1969)

3. W. von Oertzen and A. Vitturi, Rep. Prog. Phys. 64, 1247 (2001)

4. L. Corradi, et al., Phys. Rev. C 84, 034603 (2011)

5. A. Chatterjee, et al., Phys. Rev. Lett. 101, 032701 (2008) 
6. M. Evers, M. Dasgupta, D.J. Hinde, D. H. Luong, R. Rafiei, R. du Rietz, and C. Simenel, Phys. Rev. C 84, 054614 (2011)

7. E. Khan, N. Sandulescu, N. V. Giai, and M. Grasso, Phys. Rev. C 69, 014314 (2004)

8. M. Matsuo and Y. Serizawa, Phys. Rev. C 82, 024318 (2010)

9. B. Avez, C. Simenel, and Ph. Chomaz, Phys. Rev. C 78, 044318 (2008)

10. E. Khan, M. Grasso, and J. Margueron, Phys. Rev. C 80, 044328 (2009)

11. E. Pllumbi, M. Grasso, D. Beaumel, E. Khan, J. Margueron, and J. van de Wiele, Phys. Rev. C 83, 034613 (2011)

12. P.D. Kunz, program DWUCK4, University of Colorado (unpublished)

13. H. Shimoyama and M. Matsuo, Phys. Rev. C 84, 044317 (2011)

14. G. Potel, F. Barranco, F. Marini, A. Idini, E. Vigezzi, and R.A. Broglia, Phys. Rev. Lett. 107, 092501 (2011)

15. F.D. Becchetti, Jr. and G.W. Greenless, Phys. Rev. 182, 1190 (1969)

16. X. Li, C. Liang, and C. Cai, Nucl. Phys. A 789, 103 (2007)

17. M. Grasso, D. Lacroix, and A. Vitturi, Phys. Rev. C 85, 034317 (2012)

18. G. Hupin and D. Lacroix, Phys. Rev. C 83, 024317 (2011) 